\title{
Green private sector development
}

\section{Supporting small and medium enterprises towards Green Economy}

\begin{abstract}
In order for green business development services to be effective, market promotion on the supply and demand side is necessary. What is its role in the transition to Green Economy in emerging economies? Challenges and opportunities of this new concept are illustrated with experiences of German Development Cooperation from India. By Cosima Stahr and Hans Joachim Zinnkann
\end{abstract}

O ne of the objectives of German development cooperation is the strengthening of micro, small and medium-sized enterprises (MSME) in developing and transition countries. Competitive MSME represent the backbone of the economy in most developing and transition countries as this is where the vast majority of the working population is employed and earns their living - either formally or informally. By offering employment, these businesses generate income and tax revenues and thus are crucial for economic growth and a capable public sector. However, alongside their vital role in economic development, MSME also have negative impacts - labour practices often do not meet international standards and the environment suffers in the course of their economic activity. MSME are often heavy polluters as oversight over smaller-sized companies is habitualy inadequate and many work with obsolete and inefficient technology.

The objective of Germany's activities in private sector development is to strengthen MSME and their associations in partner countries, making them more competitive and thus enabling them to contribute to inclusive growth and sustainable development. Promoting trajectories towards a Green Economy in partner countries also has an immediate effect on people living in poverty, as they form the majority of MSME employees and it is their communities which are most affected by environmental pollution.

In many developing and transition countries, the private sector, and especially MSME, faces challenges regarding technology and innovation, upgrading skills and market information. German development cooperation identifies lack of existence and of access to business development services (BDS) among the key contributors of these challenges [1]. Typical providers of BDS are business associations, chambers of commerce, academic institutions, public agencies or bureaus and consultancies. Often, their technical or institutional capacities are weak.
Further, the quality of BDS offered by private or public service providers is seen as inadequate. As a result, companies in partner countries have few opportunities to upgrade their skill base and technology and continue to act on insufficient market information. The lack of BDS thus contributes to stymied economic development and weak competitiveness on global markets. This capacity gap is especially visible in environmental topics. The promotion of functioning markets for "green" i. e., environment- and climate-related - BDS is thus a central area of intervention of German development cooperation in the competence area of private sector development. The aim of Green BDS is the promotion of private sector actors such as consultants, chambers, and associations to provide market-oriented training and advisory on environmental topics to companies. Green BDS typically address fields such as pollution and environmental management, and resource efficiency. Services in these fields cover numerous topics, including environmental management systems, eco-labelling, environmental regulation, and sustainability reporting. Recently, climate change mitigation and adaptation have been added to the Green BDS agenda.

SME are most strongly affected by an absence of Green BDS, as they usually lack the capacity to develop their own approaches without external assistance. Despite factors which push businesses to become greener (e. g., regulation, resource scarcity, etc.) or pull-factors (e. g., cost savings, arising business opportunities, tax reduction), it is not assured that SME act accordingly; this is in part due to the fact that their planning horizons are usually short, and they often take a "reactive" approach, only acting when there are no alternatives. The provision of Green BDS therefore serves to enable businesses to absorb push-factors and benefit from pull-factors in a more strategic way.

Challenges for effective BDS in partner countries include absence of suitable service providers, inadequate technical and institutional capacities of existing service providers, and difficulty in making their services accessible for SME (supplyside problems). SME often have limited ability to pay for services and/or a low level of willingness to pay, because they lack awareness of the benefits these services can deliver (demandside problems).

When promoting BDS, German development cooperation seeks to be market-oriented, supporting both the supply side (BDS providers) as well as the demand side (SME). To assure that the intervention is sustainable in the long term, selected local BDS providers are supported. Ideally, while German development cooperation supports BDS providers in the devel- 
opment and implementation of Green BDS, the SME pay for these services. SME will of course only do so if the immediate business relevance of Green BDS is apparent.

To support businesses in adapting to climate change, German development cooperation in the field of green BDS promotes measures that reduce the vulnerability of businesses to changing climate and weather conditions. These include heat waves and cold waves, storms, flooding, and drought periods. Vulnerability towards longer-term trends and their impacts is also addressed - for example, the reduction of yearly rainfall, and resulting sinking water table and water scarcity.

Many climate change adaptation [2] issues have been on the agenda of businesses for a long time and have indeed played their part in Green BDS; resource efficiency, for example. One aspect of environmental management - energy efficiency - is now often framed as the key climate change mitigation issue; it is also a key topic for corporate adaptation strategies, particularly in those countries where energy supply is unreliable. A case in point was the late onset of monsoon in 2012 in India, which led to the necessity of pumping ground water in industry and agriculture in the North, thereby leading an overloading of the electric grid - resulting in a blackout that affected the entire country for days. Companies which were water- and energy efficient and/or had an alternative energy supply were at an advantage [3].

The approach to develop climate change adaptation as a topic for local BDS providers which is described below illustrates how demand-driven and market-oriented Green BDS support can be conceptualized and implemented.

\section{Experiences from India}

The India-based GIZ MSME Umbrella Programme, supported by the BMZ-financed GIZ Sector Project on Private Sector Development, together with their knowledge partner adelphi, developed climate change adaptation for SME as a topic and a capacity building approach for BDS providers.

As a first step, GIZ commissioned two studies on climate change impacts on SME, one study focusing on business risks and the other on business opportunities. As part of the former study, the most affected sectors were identified. In these sectors, which include metal working and textiles, company case studies were conducted to gather insights on the different ways in which SME are vulnerable to climate variability and climatic changes. The importance of this topic to the Indian economy became apparent in the studies. In general, Indian SME are extremely vulnerable to external shocks (such as extreme weather events) as many lack the financial capacity to deal with even short periods of production downtimes or slowdowns and are very sensitive to price hikes of material inputs. In addition, the weak infrastructure (road and railway system, electric grid, etc.) in India further increases the vulnerability of companies especially towards the end of value chains. The complexity of the issue of climate change as well as the uncertainty of its exact na- ture in the medium- and long-term were identified as hurdles for making the topic relevant to SME.

These analyses laid the basis for developing, in a second step, a capacity building approach on climate change adaptation for SME through BDS providers. Indian BDS providers' technical knowledge and expertise gaps were identified, particularly in respect to SME-focused business consultancies. The intervention aimed to close this gap and develop a capacity building approach for these BDS providers. This encompassed the development of training material and advisory services for Indian SME consultancies.

In a third step, SME-focused climate change adaptation material (called the "Climate Expert" approach) was then prepared in different formats by GIZ's knowledge partner adelphi. The material includes Training-of-Trainers to educate BDS providers, company training to be used by the BDS providers, an assessment approach on climate risks and opportunities, a consultant's manual for the provision of one-on-one advisory services, and an interactive online knowledge platform and tool (www.climate-expert.in) directed at BDS providers and SME alike. The key element of all formats is the development of a company-specific adaptation strategy in four steps: 1) a structured assessment of past and likely future impacts, 2) definition of resulting risks and opportunities, and after 3 ) a prioritization thereof, 4) the development and implementation of adaptation measures. Furthermore, qualitative and quantitative assessments of adaptation measures are part of the material. The quantitative assessment comprises a cost-benefit analysis (CBA) as well as a cost-effectiveness analysis (CEA) for adaptation measures. They allow demonstrating short- medium- and long-term analyses of payoff periods for measures concerned with resource efficiency (CBA) and a comparison between the effectiveness of different measures for those focusing on climate change risks (CEA).

In all materials, the business relevance of climate change impacts on SMEs is in focus. Climate change impacts are framed mainly as a threat to business growth and survival. The aspect of business opportunities is also part of the material they can arise from competitive advantages through adaptation, but also by product development or process innovation in response to changing climate and weather conditions. This allows SME decision makers to connect the topic of climate change to their business reality. In addition, climate change adaptation is linked with already known pressure points for businesses, including resource efficiency. This enables BDS providers to connect the adaptation-specific material with topics they have proven expertise in.

The materials were tested with SME and BDS providers and improved according to their feedback. Case studies based on company visits were developed to illustrate the training material. During the pilot trainings, additional company experiences with climate variability and extreme weather events were collected and then integrated into the materials. As a last step, local BDS providers were invited to submit proposals for busi- 
ness models on how they would market and use the capacity building materials and advisory approaches to their SME clients. In a business model and plan, they needed to demonstrate how SME would be willing and able to cover part of the costs. Out of eight submissions by local BDS providers, the best proposal received co-funding by GIZ to roll out both the trainings and the advisory services.

\section{Green BDS and climate change adaptation opening Green Economy pathways}

Promoting Green BDS for SME is a key topic for German development cooperation in the private sector, as it improves their competitiveness and therefore strengthens economic development - all the while also reducing environmental degradation and resource inefficiency which are characteristic of this sector. A key requirement of interventions of German development cooperation in this field is that services are demanddriven and market-oriented. However, demand for Green BDS from SME often needs to be strengthened. To make Green BDS relevant for SME, approaches and materials need to reflect their thinking and language. In India, assuring business survival and growth was chosen as key objective of climate change adaptation. In addition to risk management in this regard, a focus on resource efficiency (and thus cost savings) was used to create attention from SME for the topic. The material offered customised economic analytical approaches for assessing adaptation measures and corresponding investment decisions. By using business arguments, and anchoring the material in local realities through case studies and company examples, and also by using local BDS providers to market and implement the developed approaches, demand for the developed services was created.

As outlined above, ideally private and/or public actors and institutions are enabled to offer relevant market-oriented BDS on a financially sustainable basis. This is not always feasible since institutions or markets for functioning BDS are often immature in developing and transition countries [4]. Furthermore, SME in developing and transition countries often lack mid- or long-term strategic planning and thinking, which makes investment decisions, also for Green BDS, with a longer payoff time difficult. In the course of market promotion, it might be necessary to initiate the provision of certain services through donor or public funding or to even provide subsidies for some services that can be seen more as public goods. One such area seems to be services for dealing with the challenges of climate change as a resilient private sector is beneficial for the economies and societies of partner countries. The objective of Green BDS is a more efficient, more sustainable, less risk-prone and more socially equitable "Green Economy". The private sector plays a vital role in this transformation, and Green BDS are designed to support and assist businesses in the necessary changes. Through Green BDS, the requirements of development cooperation and the business reality of the private sector in developing and transition countries come into alignment. Green BDS' objectives are to assure competitiveness and make the private sector in these countries resilient to external shocks, including climate change, yet also to assist them in upgrading skills and promoting innovation, resulting in higher efficiency, less exposure to risk and greater sustainability. These are also aims that private sector actors in these countries tackle to assure competitiveness and realise market opportunities. Climate change adaptation could play a role in catalysing Green BDS.

The experience with Indian SME showed that their interest in climate change adaptation and Green BDS topics was greater when they had recently experienced an adverse climate phenomenon. Climate change dynamics could thus somewhat ironically act as an external shock that create demand for Green BDS topics, including resource efficiency and environmental management, thereby supporting Green Economy trajectories.

\section{Annotations}

[1] 'MSME' vs. 'SME': the category 'micro enterprises' in developing and transition countries often refers to 1-2 person businesses, with lowskilled entrepreneurs (some even lacking basic business skills entirely), often operating in the informal economy. This lack of entrepreneurial skills drastically limits their capacity to make use of BDS. This is especially true for the case of India which was confirmed by our experiences there. Hence, the objective to target especially MSME in the case of BDS often might need to be "reduced" to SME.

[2] The term 'climate change adaptation' is used here for adapting to climate variability and extreme weather events - it is for the moment extremely difficult to directly connect climate change dynamics to specific occurrences of either. However, both will occur more strongly and more frequently as a result of climate change.

[3] While the late monsoon onset cannot be clearly traced to climate change dynamics (though it is of course likely), a disruption of otherwise reliable systems, leading to a cascade of failures and systemic collapse is an extreme case of expected climate change impacts.

[4] The experience in India is in this regard not representative for other partner countries, as there are numerous BDS providers that cater to SME.

\section{AUTHORS + CONTACT}

Cosima Stahr is Senior Project Manager at adelphi on sustainability and companies in development cooperation, and clean tech innovation promotion. Phone: +49 30 89006880, Email: stahr@adelphi.de

Hans Joachim Zinnkann is Advisor on Green Economy \& Private Sector Development with Deutsche Gesellschaft für Internationale

Zusammenarbeit (GIZ) $\mathrm{GmbH}$ in the BMZ financed Sector Project "Innovative Approaches of Private Sector Development".

Phone: +49 6196 79-1245 Email: hansjoachim.zinnkann@giz.de

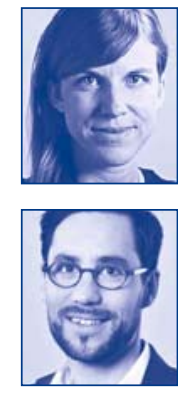

